\title{
Development of a sliding device for extended measurements in coastal waters
}

\author{
G. Zappalà ${ }^{1}$, M. Marcellì ${ }^{2} \&$ V. Piermattei ${ }^{2}$ \\ ${ }^{1}$ Istituto per l'Ambiente Marino Costiero (IAMC), \\ National Research Council, Messina, Italy \\ ${ }^{2}$ DECOS Experimental Oceanology and Marine Ecology Laboratory, \\ Tuscia University, Viterbo, Italy
}

\begin{abstract}
Extensive measurements of main sea water parameters (e.g. temperature, salinity, dissolved oxygen, nutrients, bacterial abundance, etc) are required to investigate the marine environment, both to evaluate its state and to quickly detect possible perturbations (arrival of pollutants, anthropogenic contaminants, etc).

Traditional observing methods cannot offer the flexibility and cost effectiveness needed for extensive monitoring and water quality assessment in coastal areas where important health and economic issues are involved (e.g. aquaculture, tourism).

As part of the MFSTEP program (EU FP5) a new device was designed and built for pelagic use, the SAVE (Sliding Advanced VEhicle), able to perform continuous profiles of physical and optical measurements on the upper $200 \mathrm{~m}$ of the water column.

The original system consists mainly of a depressor, towed at a fixed depth, using a cable on which the main unit slides. Both the depressor and the sliding unit can be equipped with various kinds of sensors.

The new goal was to obtain a coastal version, to be towed from small boats.

A smaller prototype is now under development and testing, and it is able to be towed by a very small $(8-10 \mathrm{~m})$ boat, like those commonly used in aquaculture plants, and also fitted with modular water samplers for bacterial or chemical determinations.
\end{abstract}

The paper gives some first results of this development.

Keywords: operational oceanography, coastal monitoring, water sampling. 


\section{Introduction}

Satellite remote sensing provides a unique synoptic view of phenomena such as phytoplankton biomass concentration and sea surface temperature, but the measurement validation requires constant effort and as much sea-truth data as possible, mainly for biological variables, but also for physical ones; for this reason the integration between in situ measurements and remote sensing techniques should be improved also for the development of ecosystem model forecasts.

Operational oceanography finds in the synoptic observations the best tool to study marine variables, which are investigated and collected through remote sensing; for this reason, these observations need to be validated with in situ data, possibly collected and transmitted in real time.

At the end of the past century, the Authors designed and tested various vehicles to perform coastal and offshore monitoring (T-FISH [1], Sarago, [2]), that needed to be towed by a 15-20 m. vessel.

The innovative platform shown in this work is the upgrade and improvement of a sliding vehicle, called SAVE, designed and built during the MFSTEP Project, and partly inspired to a vehicle described by Nomoto et al. [3].

This advanced platform allows to perform a detailed physical and biological characterization of the water column till $200 \mathrm{~m}$. depth.

The new model is designed to be used on little boats for coastal monitoring.

\section{The SAVE prototype}

During the MFSTEP (Mediterranean Forecasting System: Toward Environmental Prediction) Project, the European Community funded a technological development program. The aim was to design and build a new sliding vehicle for oceanographic research.

The product of this project was a prototype called SAVE (Sliding Advanced VEhicle), conceived to be modular, flexible and versatile.

SAVE was designed to carry out detailed and rapid profiles, in order to have quasi-synoptic measures of physical and biological variables. The system permits to perform a detailed characterization of the water column, following the dynamic evolution of natural phenomena.

It consists in one on-board and one underwater unit, able to manage various instrumental payloads.

The underwater unit comprises a depressor, towed at a fixed depth by a surface unit and an instrumented vehicle, sliding along a cable from the surface to the bottom [4].

The depressor is equipped with inclinometers for pitch and roll and pressure transducer in order to monitor the behaviour of the unit during the operation.

The vehicle can also house sensors for physical and biological parameters.

The design involved a long study period, because of the analysis of the single system components, that was performed through a navigation model. The model took into account different shapes, weights, materials, cables and many other 
components, which influence the behaviour of the system in the water column at the different operating conditions.

All the forces applied to the system were analysed and the behaviour of the system at different towing speeds was simulated.

A special attention was given to the definition of the geometry of the depressor and of the hydrodynamic control surfaces of the SAVE vehicle and of their moving, in order to obtain the expected performances at the different operating conditions.

The comparison between different NACA profiles efficiency brought us to chose a particular wing geometry for the depressor.

The selected profile was NACA 4412, because of the good ratio between lift (CL) and drag (CD) coefficients, also with high operational angles.

In order to improve the profile performance two extremity plates were added, reducing the fluid motions between the overpressure margin and the depression margin, as shown in Fig.1.

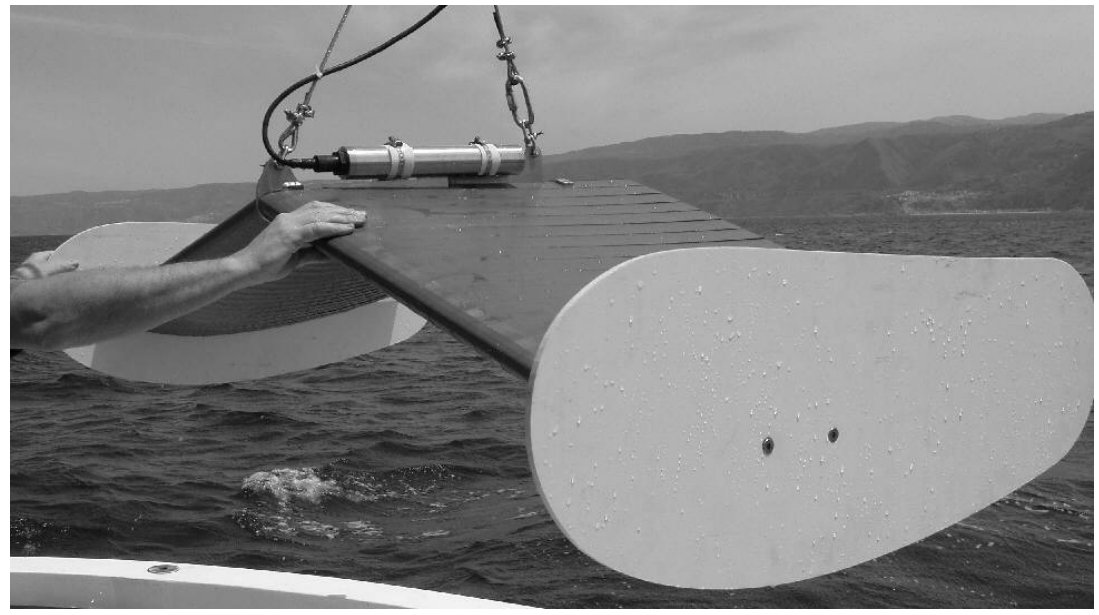

Figure 1: $\quad$ First depressor configuration.

The SAVE vehicle is constituted by an instrumented pressure cylinder; the guide system is located on the front side of the vehicle and is composed by pulleys connected to the cable, as reported in Fig. 2.

The final configuration was designed to operate at a maximum $200 \mathrm{~m}$ depth, with a speed of 2-8 knots. The first depressor was built in PVC, with an internal steel structure to join together the ribs composing the wing and was tested during various cruises.

The behaviour of the depressor was monitored using a dedicated data acquisition board ( $1 \mathrm{~Hz}$ sampling rate), interfaced with a miniature pressure transducer (0 to $500 \mathrm{dBar}$ range, $0.1 \mathrm{dBar}$ resolution) and a biaxial inclinometer (Roll and Pitch angle range \pm 100 degrees, 1 degree angular resolution); the board, able to store up to 4000 samples and to transmit them serially, is shown in Fig. 3. 


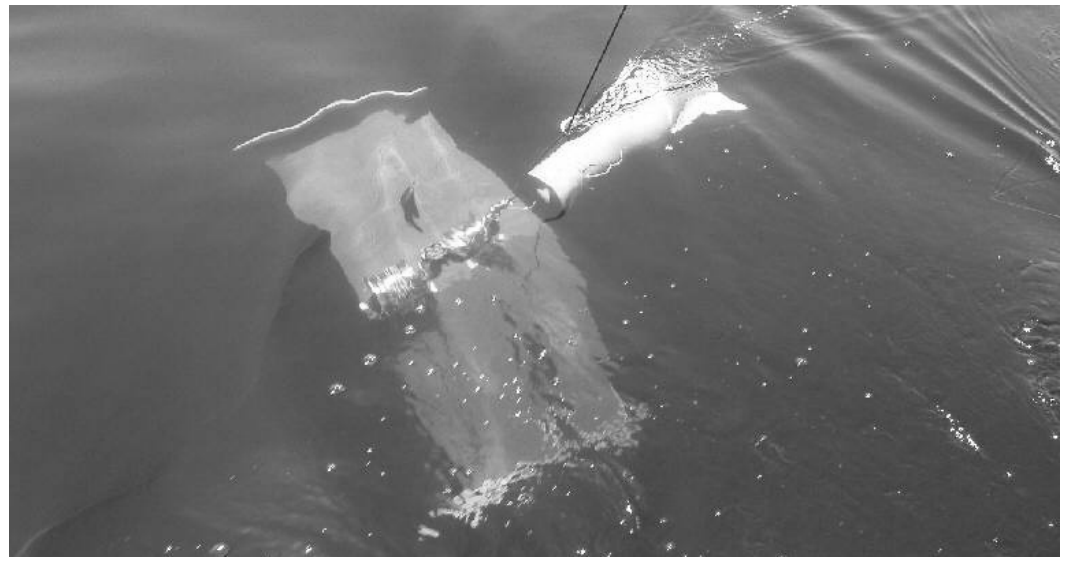

Figure 2: $\quad$ Field tests of SAVE depressor and vehicle.

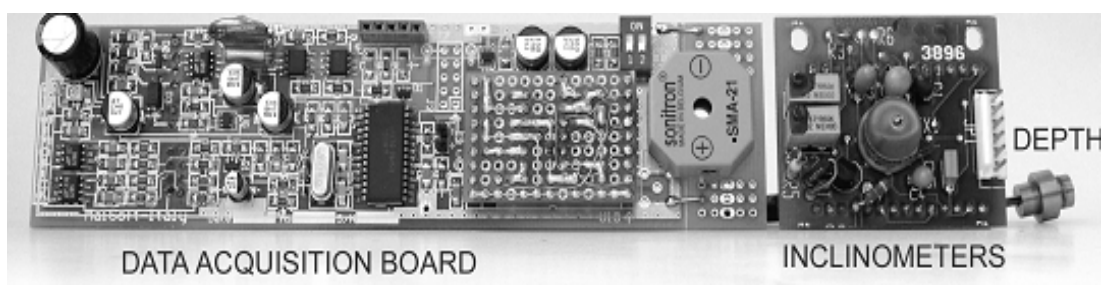

Figure 3: The wing attitude monitoring electronics.

The board and the sensors were inserted into a cylindrical pressure hull installed on the depressor itself, as shown in Fig. 4.

Tests were performed in Messina towing the depressor at a fixed depth of about 31 meters; results are shown in Fig. 5 and highlight the high stability of the depressor.

\section{The new model}

A further cooperation between the Laboratory of Experimental Oceanology and Marine Ecology of DECOS (Tuscia University) and CNR-IAMC Section of Messina started to improve previous instrumentation in order to design and build a new coastal monitoring system. The main characteristics are little dimensions and ease of use on little boats.

For this purpose some changes were carried out, the first concerning the depressor configuration.

In order to facilitate the construction of the depressor, a straight wing $\left(\varphi=0^{\circ}\right)$ was designed, housing a recess for the electronic components, as shown in Fig.6; this case withstands external pressure and hosts the pressure transducer and inclinometers, allowing to spare the external cylinder and improving the depressor behaviour. 


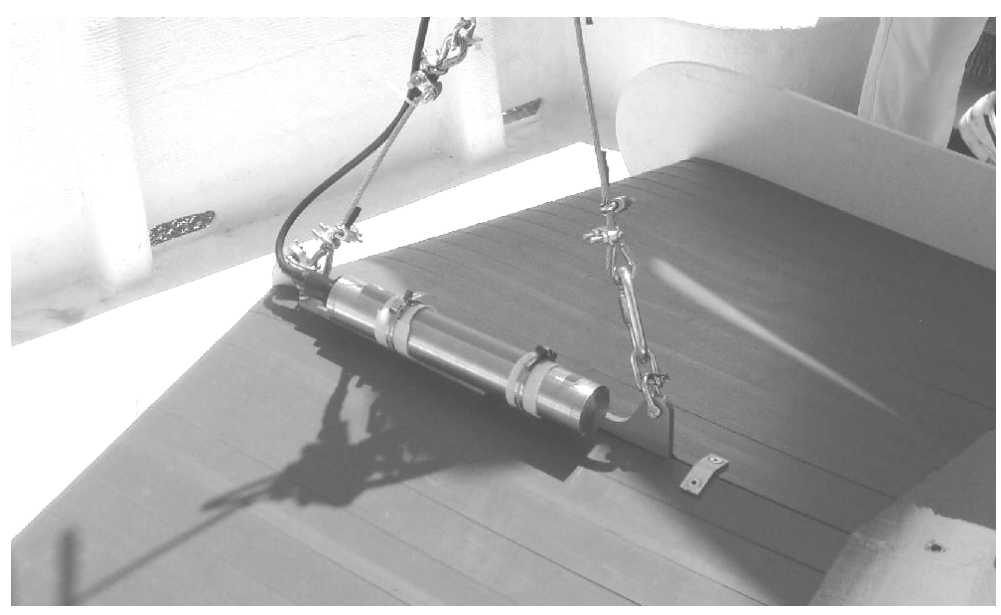

Figure 4: Instrumented pressure hull installed on the depressor.
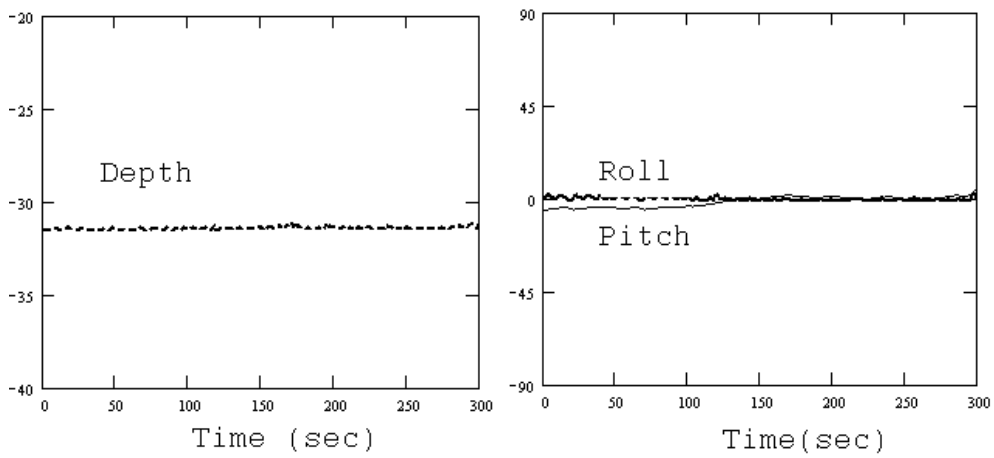

Figure 5: Depth in metres (left) and Roll and Pitch in degrees (right) measured during the first tests.

\subsection{Main characteristics of depressor sensors}

\subsubsection{Pressure transducer}

The depth measure is carried out by a 50 Bar pressure transducer, whose signal is amplified and then filtered using a $12 \mathrm{~dB}$ /octave Butterworth low-pass filter to remove the noise caused by the water flow on the membrane.

\subsubsection{Inclinometers}

The measure of the rotation components on longitudinal and transversal axes, roll and pitch, is carried out by a liquid electrolyte biaxial inclinometer $\left( \pm 60^{\circ}\right)$. The biaxial sensor is connected to a special signal conditioner, generating the signals concerning the two rotation axes. 
A wing-depressor was built to scale to carry out the first hydrodynamic tests, as shown in Fig.7.
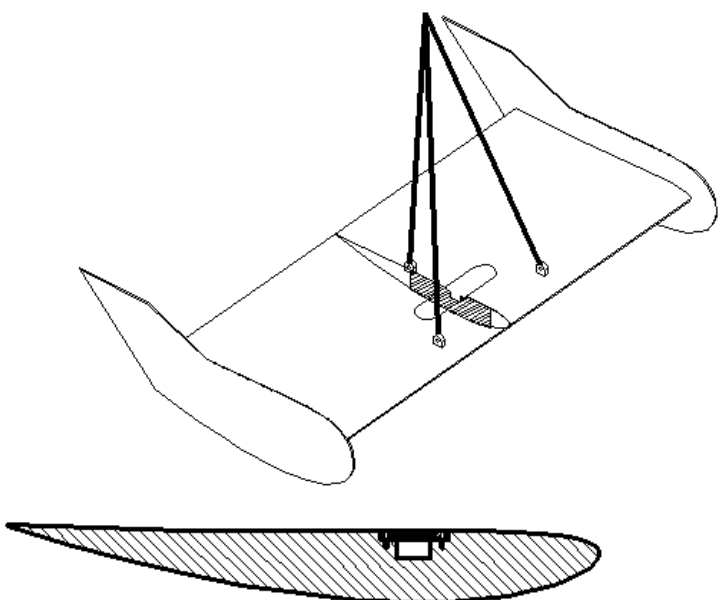

Figure 6: New depressor configuration, with internal electronics housing.
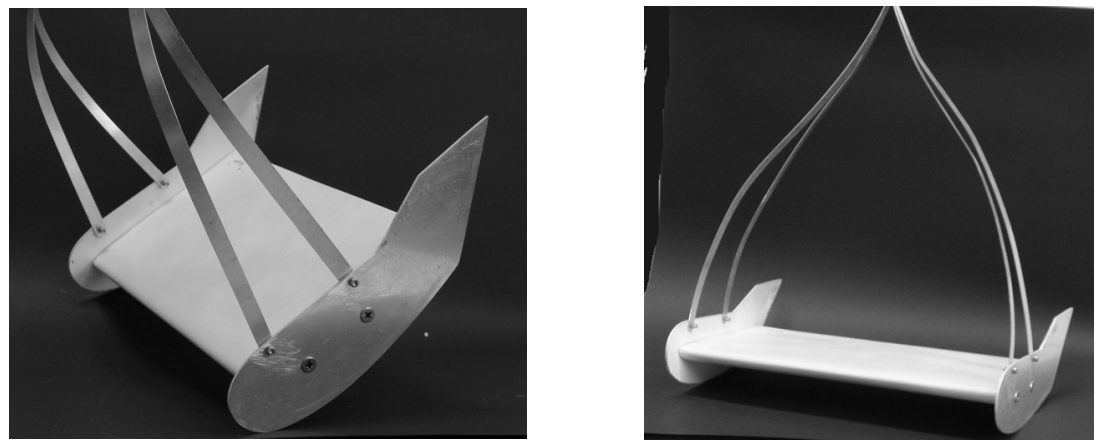

Figure 7: New depressor built to scale.

The first new model offers an instrumented vehicle towed at fixed depth, able to perform continuous profiles of chlorophyll a, temperature and conductivity along the water column.

\subsection{Cable and winch}

The cable is one of the main elements of the system not only because it sustains the weight and the hydrodynamic drag of the depressor, but also because it can constitute a resistance and a perturbation to its movement. 
The behaviour of the cable in the water column was studied by means of numerical models, in order to analyse the dynamic balances with different operative conditions, like towing speed, cable length, weight, drag.

The best results were obtained with a $9 \mathrm{~mm}$ electromechanical cable in zincplated steel, containing 133 leads covered with RILSAN.

An ad hoc winch was designed to manage the selected cable. It is moved by an electric motor $(24 \mathrm{~V} 800 \mathrm{~W})$, and is equipped with a mechanical disk brake to control descent and ascent speed.

\subsection{Water samplers}

Previous experience in the design of water samplers [5] was used to produce and test the new prototypes, the first of which, obtained modifying a device designed and used in another research program, is shown in Fig. 8.

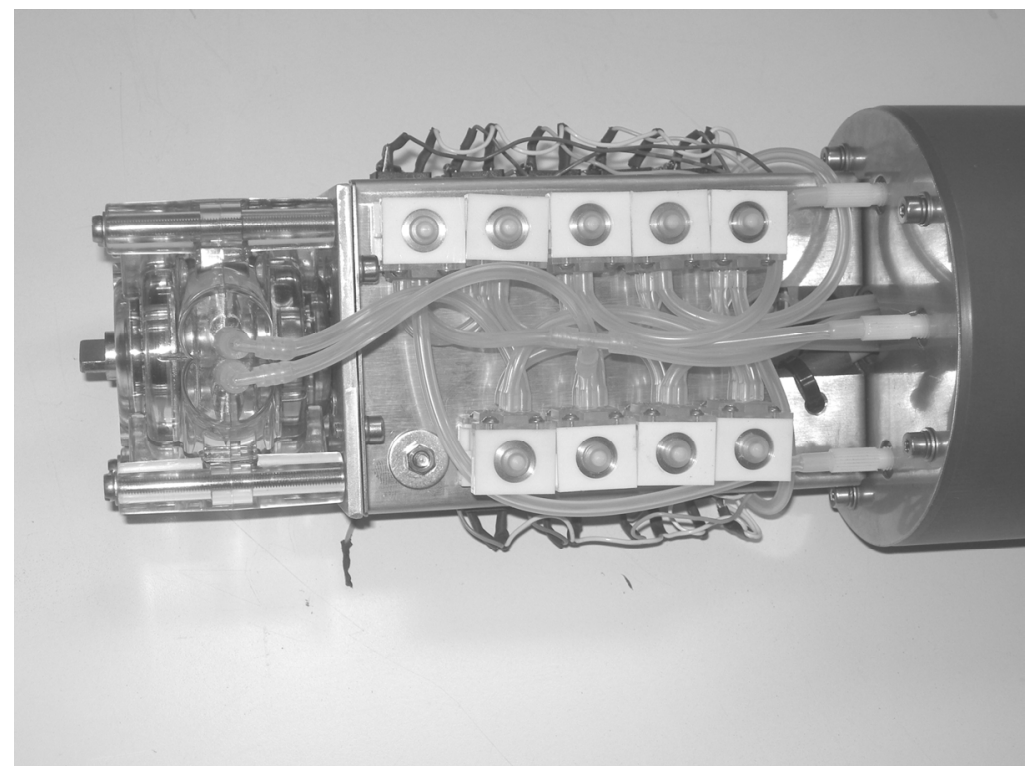

Figure 8: A close-up view of a first prototype of water sampler.

Three kind of water samplers were designed and are under test.

The first one uses a peristaltic pump to fill up to eight $250 \mathrm{ml}$ bags connected to a eight electro-valves manifold; it is possible to use another pump to add a preservant solution (usually formalin) or have it pre-dosed in the bags.

The second one uses a peristaltic pump to fill up to eight $50 \mathrm{ml}$ wells, without addition of preservant.

The third solution uses no pumps; each sampling container has one waterfilling pipe and one air-exhausting pipe, kept usually closed by pinch-type electro-valves, that, when opened, permit the water to enter the container, evacuating the air from it. 
These sample containers might be disposable bottles or cavities drilled in a plastic cylinder, but in both cases they must be able to withstand the external water pressure, being immersed empty and close.

A low power microcontroller based electronics manages all the operations, interfacing with the depressor electronics via RS232. Commands are issued by the control system on board the towing vessel; sampling positions are preprogrammed and geo-referenced using a system derived from that used in the automatic multiple launcher for expendable oceanographic probes developed in the already cited MFSTEP project [6].

\section{Conclusions}

The new developed technology reported in this work answers to the claim of high resolution system for a synoptic study of marine ecosystem, which is necessary due to the high space and time variability of its phenomena.

The described devices offer high versatility and reliability at a relatively low cost, and could constitute the core for new coastal monitoring systems.

\section{Acknowledgements}

The authors acknowledge the funding support received by Italian and European research programs, and thank their responsibles: Nadia Pinardi, Giuseppe Manzella (MFSTEP), Ermanno Crisafi (SAM), Alessandro Bergamasco, Simone Mirto (PON Acquaculture Cages).

\section{References}

[1] Crisafi, E., Azzaro, F., Zappalà, G. \& Magazzù G., Integrated automatic systems for oceanographic research: some applications. Proc. of OES-IEEE OCEANS '94 Conference, I, pp. 455-460, 1994.

[2] Marcelli, M. \& Fresi, E., The Sarago Project. Sea Technology, pp 62-67, 1997.

[3] Nomoto, M., Tsuji, Y., Misumi, A. \& Emura, T., An advanced underwater towed vehicle for oceanographic measurements. Advances in underwater technology, ocean science and offshore engineering, 6, pp. 70-88, 1986.

[4] Marcelli, M., Di Maio, A., Piermattei, V., Zappalà, G. \& Manzella, G., Development of new technologies for the high variabilità phenomena data acquisition in the MFSTEP-VOS project. European Operational Oceanography: Present and Future, Proc. of the Fourth International Conference on EuroGOOS, eds. H. Dahlin, N.C. Flemming, P. Marchand, S. E. Petersson, pp. 184-187, 2006.

[5] Zappalà, G., Caruso, G., Crisafi, E., Coastal pollution monitoring by an automatic multisampler coupled with a fluorescent antibody assay. Proc. Of the 5th Int. Conf. on Environmental problems in coastal regions, Coastal Environment V, eds. C.A. Brebbia, J. M. Saval Perez, L.Garcia Andion \& Y Villacampa, WIT Press, Southampton (UK), pp. 125-133, 2004. 
[6] Zappalà, G., Reseghetti, F., Manzella, G.M.R., Development of an automatic multiple launcher for expendable probes, Ocean Sci., 3, pp. 173178, 2007. Online www.ocean-sci.net/3/173/2007/ 